\title{
Quality Indicators in Pancreatic Surgery: Lessons Learned from the German DGAV StuDoQIPancreas Registry
}

\author{
Ulrich F. Wellner ${ }^{a}$ b Tobias Keck ${ }^{b}$ \\ ${ }^{a}$ German Society for General and Visceral Surgery (DGAV), Berlin, Germany; \\ ${ }^{b}$ Clinic for Surgery, University Clinic Schleswig-Holstein Campus Lübeck, Lübeck, Germany
}

Keywords

Quality indicator - Pancreatic surgery · Risk factor .

Clinical registry

\section{Summary}

Background: Political and public interest in quality management in surgery is increasing. The German Society for General and Visceral Surgery (DGAV) established the DGAV StuDoQ, a nationwide registry for quality assessment in visceral surgery, with the organ-specific module DGAV StuDoQIPancreas. The first prerequisite for the measurement of quality is the definition of quality indicators. These can be related to risk factors which are also documented in the registry. Methods: Quality indicators for pancreatic surgery were developed by review of the current literature and expert consensus. After ranking the potential quality indicators, three essential indicators for outcome quality were selected for further review of the literature. Current figures were extracted from the DGAV StuDoQIPancreas registry and the correlation with selected risk factors was tested. Results: Three essential outcome quality indicators were selected: in-hospital mortality, TV30, and severe complications according to the Clavien-Dindo Classification. Preliminary data confirms the validity of risk factors included in the DGAV StuDoQIPancreas registry. Conclusion: Essential quality indicators were defined for pancreatic surgery. The DGAV StuDoQIPancreas constitutes a valid platform for risk-adjusted quality assessment in Germany.

(c) 2017 S. Karger GmbH, Freiburg

\section{Introduction}

In view of the increasing public and political interest in quality assurance in the health care system, the German Society for General and Visceral Surgery (DGAV) created a platform for the assessment of quality in visceral surgery, i.e. the DGAV StuDoQ. The StuDoQ|Pancreas was developed as the organ-specific module (Wellner UF; unpublished data). As an essential element, StuDoQ|Pancreas does not only contain parameters for surgical technique and the postoperative course but also detailed information on known risk factors in pancreatic surgery. This results in the possibility to measure and evaluate not only quality indicators (QI) on the basis of the registry but also i) to examine the influence of risk factors on the QI and ii) to adjust QI measurement to the risk profile of patients or institutions.

Quality in the health care system can be divided into the aspects of structure, process, and result quality [1]. QI do not measure quality directly but function as surrogate parameters, i.e. measures which make a distinction between good and poor quality. As such, they should meet the requirements of the following categories according to the so-called QUALIFY criteria: relevance, scientific evidence, and practicability. Relevance results from the relevance and usefulness to health care; scientific evidence is derived from clarity of definition and published scientific data; and practicability is a question of intelligibility for professional staff, patients, and the public, availability and verifiability of data as well as the expenditure effort. Since individual QI as surrogate parameters only partially represent the quality to be measured, the use of multiple QI as QI profiles is recommended.

The current literature provides little data on the definition and recording of quality indicators in pancreatic surgery. Here, we review QI in pancreatic surgery and provide current data from the DGAV StuDoQ|Pancreas registry.

\section{KARGER}

() 2017 S. Karger GmbH, Freiburg
PD Dr. med. Ulrich F. Wellner 
Table 1. Search terms for literature review

\begin{tabular}{|c|c|}
\hline Topic & Search term \\
\hline $\begin{array}{l}\text { Quality indicators for } \\
\text { pancreatic surgery }\end{array}$ & $\begin{array}{l}\text { (quality indicators pancreatic surgery) OR (quality indicators pancreatec- } \\
\text { tomy) OR (quality indicators pancreatic resection) }\end{array}$ \\
\hline Perioperative mortality & $\begin{array}{l}\text { (English [Language] OR German [Language]) AND (“2005/01/01" [PDAT]: } \\
\text { "10y" [PDAT]) AND ((“"pancreatectomy" [MeSH Terms] OR "pancreati- } \\
\text { coduodenectomy" [MeSH Terms] OR "pancreaticojejunostomy" [Title / Ab- } \\
\text { stract] OR "pancreaticoduodenectomy" [Title / Abstract] OR "pancreaticoje- } \\
\text { junostomy" [Title / Abstract] OR "pancreaticojejunostomy" Abstract] OR } \\
\text { "pancreatoduodenectomy" [Title / Abstract] OR "pancreatojejunostomy" } \\
\text { [Title / Abstract] OR ("pancreatic" [Title / Abstract] AND ("resection" [Title } \\
\text { / Abstract] OR "surgery" [Title / Abstract]) )) AND "mortality" [Title / Ab- } \\
\text { stract] AND ("prospective" [Title / Abstract] OR "population-based" [Title / } \\
\text { Abstract] OR "randomized" [Title / Abstract] OR "randomized" [Title / Ab- } \\
\text { stract] }\end{array}$ \\
\hline $\begin{array}{l}\text { Clavien-Dindo } \\
\text { classification }\end{array}$ & $\begin{array}{l}\text { Clavien Dindo AND (pancreatectomy OR pancreatoduodenectomy OR pan- } \\
\text { creatic surgery OR pancreatic resection) AND (OR prospective randomized } \\
\text { OR population based) }\end{array}$ \\
\hline
\end{tabular}

\section{Material and Methods}

\section{Literature Search}

For the identification of relevant publications, a search with the terms listed in table 1 was conducted in the PubMed database (September 2015). Only population-based and prospective studies of the last 10 years with an English abstract for further assessment were included in the review.

\section{Assessment of Quality Indicators}

The obtained QI from the literature search were summarized and evaluated by an expert panel according to the guidelines of the Agency for Quality in Medicine with regard to relevance to the health care system, clarity of definition, changeability, scientific evidence, and risk of misdirection [1]. Thereafter, the expert panel sought agreement for the selection of a few QI which were regarded as most essential (essential QI), and the rationale for selection was documented. The literature was then screened for reference values of the essential QI (the search terms are given in table 1). Current values were calculated from the StuDoQ|Pancreas registry using the 'metafor' package of the $\mathrm{R}$ software [2] with the parameters outlined below.

\section{Results}

\section{Literature Review}

Out of 144 publications featuring the search term, only two explicitly dealt with the topic of QI. Bilimoria et al. [3] established a panel of 29 QI with a high degree of validity in an expert panel (American College of Surgeons' Quality Indicator Development Expert Panel) through a structured review process of the literature. Reference values are only given for a few QI. Based on a national survey of pancreatic surgeons in the USA, Kalish et al. [4] developed a list of 12 relevant QI. Reference values for the QI were not specified. The current German S3 guideline for exocrine pancreatic carcinoma [5] lists 5 QI with reference values: rate of R0 resections (target $\geq 70 \%$ ), lymph node yield (target $\geq 10$ ), completeness of the pathology findings (goal 100\%), adjuvant therapy with existing indication (target $\geq 50 \%$ ), and palliative therapy with existing indication (target 100\%). The NCCN (National Comprehensive Cancer Networks) Guidelines for Pancreatic Adenocarcinoma of the USA do not mention QI [6].

\section{Evaluation of Quality Indicators}

With regard to result quality the following QI were scored in descending order: mortality, readmission, bleeding, severity of complications, overall complications, postoperative pancreatic fistula, lymph node yield, patient-reported outcomes, and R0 resection. Concerning process quality, the following ranking of the best 10 QI could be established: preoperative cross-sectional imaging, perioperative antibiotic treatment, thromboprophylaxis, resection of pancreatic cancer in stage 1 and 2, the time of initial diagnosis to therapy, no resection of pancreatic cancer at stage 4, detailed preoperative risk assessment, standard lymphadenectomy, implementation of adjuvant and neoadjuvant therapy with existing indication. As the main parameters of structural quality, the availability of interventional radiology and an intensive care unit with specially trained personnel, the institutional and individual caseload, interdisciplinary treatment, and certification of surgeons were evaluated.

\section{Agreement on Essential Quality Indicators}

Among the highest rated QI, the following were assessed as essential in the expert consensus: perioperative mortality, postoperative hospital stay, major complications according to the ClavienDindo Classification (CDC). Herein, the composite endpoint TV30 was suggested (see below). Regarding structural quality, the permanently ( $24 \mathrm{~h} / 7$ days) available interventional radiology was assessed as essential. The rationales and literature reference values are set out as follows. 
Rationale for Perioperative Mortality as a Quality Indicator

Among the complex surgical procedures, pancreatic resections take a special place because, on the one hand, they are among the interventions with the highest mortality and, on the other hand, have the highest volume-outcome correlation [7]. According to current routine data, perioperative mortality of pancreatic resections is about $10 \%$ in Germany [8]. This is in quite sharp contrast to the value of $\leq 5 \%$ reported as a benchmark in the international literature [7]. As one of the most important influencing factors, the institutional [7] and more rarely the individual caseload of the surgeon $[9,10]$ has been repeatedly highlighted. In Germany, this has been taken into account by means of a minimal caseload requirement since more than 10 years. However, the effectiveness of the regulation in the sense of increasing centralization and reduction of mortality, following the example of other countries, has not been evaluated [11-13].

\section{Reference Values for Perioperative Mortality}

Most authors consider 5\% as the upper limit for pancreatic surgery at centers [7]. According to current routine data, however, the overall mortality in Germany is approximately $10 \%[8,14]$. The recently conducted multicenter randomized trial RECOPANC on pancreatoduodenectomy also showed a mortality of just over $5 \%$, although participants were academic high-volume centers for pancreatic surgery [15].

The use of 90-day mortality has been established for pancreatic surgery because it has been shown to be more reliable in perioperative mortality than 30-day or in-hospital mortality [16]. The latter is strongly influenced by the financial structure of the health care system. Hospital stay reported by North American centers is usually much shorter than that reported in Germany [15], while readmission rates are higher. It is therefore assumed that the difference between in-hospital mortality and 90-day mortality is minimal in Germany, which is confirmed by two recent studies with a high level of evidence $[14,15]$. In addition, the cost of a routine full coverage of 90 -day mortality is estimated to be too high to match practicability as most of the patients have already been discharged.

Depending on the extent of pancreatic resection, mortality is subject to variations. In a recent complete survey of pancreatic resections in Germany [8], in-hospital mortality differs between 7 and $23 \%$ depending on the extent of pancreatic resections. This confirms the meta-analysis of the current international literature [7]. As already outlined, there is also a strong volume-outcome relationship in pancreatic surgery. This effect is stronger than in all other areas of surgery [7]. Data concerning volume-outcome relationship in Germany remain scarce [14].

The DGAV StuDoQ|Pancreas registry was screened for in-hospital mortality of elective pancreatoduodenectomy in the past 3 years. In-house mortality was $3.8 \%$ (95\% confidence interval (CI): 2.6-5.5\%) (table 2). This result is compatible with data reported from international centers and may be interpreted, on the one hand, as a hint towards selection of institutions with high expertise and, on the other hand, as proof that a low mortality can be reached at centers.
Table 2. Evaluation of essential quality indicators from the DGAV StuDoQ|Pancreas registry; confidence intervals (CI) derived from randomeffects meta-analysis across participating centers

\begin{tabular}{ll}
\hline $\begin{array}{l}\text { Cohort } \\
\text { Index operation }\end{array}$ & $\begin{array}{l}\text { pancreatoduodenectomy } \\
\text { only validated data; elective operation } \\
09 / 2013-08 / 2016 \text { (36 months) }\end{array}$ \\
Period & 1,591 \\
N & \\
\hline Quality indicators & $3.8 \%$ \\
In-hospital mortality & $95 \%$ CI: $2.6-5.5 \%$ \\
& $20 \%$ \\
TV30 & $95 \%$ CI: $17-23 \%$ \\
& $16 \%$ \\
CDC grade 3b and 4 & $95 \%$ CI: $14-19 \%$ \\
\hline
\end{tabular}

Table 3. Simplified criteria of the Clavien-Dindo Classification (CDC) of complications in surgery; simplified according to [22]

\begin{tabular}{ll}
\hline CDC degree & Criteria \\
\hline 1 & no need of specific drug or invasive treatment \\
\hline 2 & $\begin{array}{l}\text { need for specific medical treatment eyelets } \\
\text { (e.g. transfusion, parenteral nutrition) }\end{array}$ \\
\hline $3 \mathrm{a}$ & $\begin{array}{l}\text { invasive diagnostics / therapy without general anesthesia } \\
\text { invasive diagnostics / therapy with general anesthesia }\end{array}$ \\
\hline $4 \mathrm{~b}$ & $\begin{array}{l}\text { single organ failure } \\
\text { multiorgan failure }\end{array}$ \\
\hline 5 & death \\
\hline
\end{tabular}

\section{Rationale for TV30 as a Quality Indicator}

The so-called TV30 indicator is a composite endpoint based on postoperative death, transfer to another acute care clinic, or length of stay $>30$ days after surgery. The postoperative hospital stay is considered as a good surrogate parameter for any kind of complications. This is confirmed by the current literature: In a current meta-analysis, which includes population-related data from 11 countries, a significant correlation of hospital stay with morbidity, mortality and risk factors has been demonstrated [7]. As early perioperative mortality and transfer to another institution can influence the length of stay as confounders, these parameters were combined with length of stay in the TV30. The TV30 is already successfully used as a QI for certification purposes within the framework of the DGAV.

\section{Reference Values for TV30}

With regard to length of stay, no valid reference value for Germany can be derived from the international literature: In the most recent randomized trials of pancreatoduodenectomy from Germany (RECOPANC and PANDRA) [15, 17], for example, hospital stay after pancreatoduodenectomy is more than twice as long as that reported by US centers (median 16 days, average 19 days). The DGAV StuDoQ|Pancreas registry shows a median hospital stay of 15 days, with a distribution of the 90th and 95th percentile at 33 and 44 days, for elective pancreatoduodenectomy during the past 3 years. As to the TV30 parameter in pancreatic surgery, no data 
Table 4. Ranking of quality indicators for pancreatic surgery; scoring according to the guideline for quality indicator development [1]

\begin{tabular}{lll}
\hline Parameters & Score & Category \\
\hline Mortality & 13 & Outcome \\
Readmission & 12 & Outcome \\
Postpancreatectomy hemorrhage & 11.75 & Outcome \\
Complication severity / major complications & 11.5 & Outcome \\
Overall complications & 10.75 & Outcome \\
Postoperative pancreatic fistula & 10.75 & Outcome \\
Lymph node yield & 9.25 & Outcome \\
Patient-reported outcome & 7.75 & Outcome \\
Margin-negative resection & 7.25 & Outcome \\
Preoperative cross-sectional imaging and assessment & 15 & Process \\
Perioperative antibiotics & 15 & Process \\
Venous thromboembolism prophylaxis & 15 & Process \\
Resection for stage I/II pancreatic cancer & 13.5 & Process \\
Time from diagnosis to therapy & 11.75 & Process \\
No resection for stage IV pancreatic cancer & 11.5 & Process \\
Detailed preoperative risk assessment & 11.5 & Process \\
Lymphadenectomy: standard procedure and reporting & 11.5 & Process \\
Adjuvant therapy & 11 & Process \\
Neoadjuvant therapy & 10.5 & Process \\
Standard pathology workup protocol & 10.5 & Process \\
Completeness of pathology report & 10.5 & Process \\
Endoscopic retrograde drainage & 10.5 & Process \\
Palliative therapy & 9 & Process \\
Frozen section for suspicious lymph nodes outside of standard resection area & 9 & Process \\
Documentation of TNM clinical stage for non-resected patients & 9 & Process \\
Establishment of individual treatment plan & 8.75 & Process \\
Appropriate timing of adjuvant therapy & 8.5 & Process \\
Interventional radiology available on-site & 15 & Structure \\
Intensive care unit with specialized staff on-site & 13.25 & Structure \\
Surgeon volume & 12.25 & Structure \\
Institutional volume & 12 & Structure \\
Multidisciplinary treatment & 12 & Structure \\
Surgeon certification & 10.25 & Structure \\
\hline & & \\
\end{tabular}

have yet been published. The DGAV StuDoQ|Pancreas registry shows a relative frequency of $20 \%$ (QI 17-23\%) for elective pancreatoduodenectomy over the past 3 years (table 2).

\section{Rationale for Major Complications according to the}

\section{Clavien-Dindo Classification as Quality Indicator}

The graduation of complications according to the CDC is an international standard which is regarded as a valid QI (table 3). The standardized assessment of the degree of complication is considered to be useful, but the detailed breakdown into all CDC subcategories opposes practicability. In the literature, grades $3 \mathrm{~b}$ and higher are usually summarized as major complications. Grade $3 \mathrm{~b}$ essentially means intervention with necessity of general anesthesia. CDC grade 4 applies when single organ dysfunction (4a) or multiorgan dysfunction (4b) occurs or intermediate or intensive care unit treatment is required.

\section{Reference Values for Major Complications according to the Clavien-Dindo Classification}

In the literature, there are few current studies with a high level of evidence (population-based or randomized) which report the complications according to the CDC. Furthermore, only one study reports CDC $\geq 4 \mathrm{a}$ as $7 \%$ [18]. Screening of the StuDoQ|Pancreas registry shows a relative frequency of CDC grade $3 \mathrm{~b}$ and 4 complications of $16 \%$ (95\% CI: $14-19 \%)$.

\section{Interventional Radiology as a Quality Indicator}

The importance of interventional radiology for the treatment of complications in pancreatic surgery is undisputed. Interventional drainage of intra-abdominal abscesses and interventional hemostasis of arrosion bleeding can often avoid complicated reoperations. Although this is generally accepted by pancreatic surgeons, the literature, and in particular the evidence, is very thin [19-21]. Nevertheless, this parameter of structural quality is regarded as essential by the aforementioned expert panel.

\section{Further Possible Quality Indicators}

For reasons of practicability, only a few QI were considered as essential. Other QI-related QI are listed in table 4. All QI can be recorded via the DGAV StuDoQ|Pancreas registry for comparison and validation purposes. 
Table 5. Selected risk factors with significant correlation to in-hospital mortality in the DGAV StuDoQ|Pancreas registry

\begin{tabular}{|c|c|}
\hline Category & Parameter \\
\hline $\begin{array}{l}\text { Preoperative risk } \\
\text { factors }\end{array}$ & $\begin{array}{l}\text { higher age } \\
\text { higher body mass index } \\
\text { elevated serum creatinine } \\
\text { history of myocardial infarction } \\
\text { higher ASA category } \\
\text { ascites } \\
\text { diabetes mellitus } \\
\text { esophageal varices } \\
\text { terminal renal failure (hemodialysis) } \\
\text { liver cirrhosis } \\
\text { need of care } \\
\text { jaundice }\end{array}$ \\
\hline $\begin{array}{l}\text { Intraoperative risk } \\
\text { factors }\end{array}$ & $\begin{array}{l}\text { longer operating time } \\
\text { arterial resection } \\
\text { colon resection } \\
\text { soft pancreatic texture } \\
\text { intraoperative blood transfusion }\end{array}$ \\
\hline
\end{tabular}

Validation of Risk Factors in the DGAV StuDoQ|Pancreas Registry

In the development of the StuDoQ $\mid$ Pancreas registry, major risk factors for perioperative complications were included a priori as parameters (Wellner UF; unpublished data) on the basis of the current literature in order to enable future risk adjustment of QI assessment. For a preliminary validation of the risk factors, the correlation of in-house mortality with risk factors was tested. The re- sults show significant correlations of several risk factors with hospital mortality (table 5). Both pre- and intraoperative risk factors are represented.

\section{Conclusion and Outlook}

On the basis of the current literature, QI for pancreatic surgery were evaluated and selected by expert consensus. The following QI for outcome quality ranked high and were considered as essential QI: hospital mortality, TV30, and rate of major complications according to the CDC. Regarding structural quality, permanent availability of interventional radiology is considered mandatory. The international literature is only in part suitable for defining reference values for these QI in Germany. Current data from the DGAV StuDoQ|Pancreas registry is presented which might form the basis for reference values for practice in Germany. The DGAV StuDoQ|Pancreas registry provides a perspective for a risk-adjusted quality assessment in pancreatic surgery. However, statistical considerations suggest that such calculations depend on a high case number for validity. These case numbers will be reached soon for risk adjustment to be established in the DGAV StuDoQ|Pancreas registry.

\section{Disclosure Statement}

All authors declare that there are no potential conflicts of interests in relation with this article.

\section{References}

1 Ärztliches Zentrum für Qualität in der Medizin (ÄZQ): Manual Qualitätsindikatoren. (äzq Schriftenreihe.) Neukirchen, 2009. www.aezq.de/mdb/literatur/ manual-qualitaetsindikatoren (last accessed: 13.8.2016).

2 Viechtbauer W: Conducting meta-analyses in R with the metafor package. J Stat Softw 2010;DOI: 10.18637/ jss.v036.i03. https://www.jstatsoft.org/article/view/v036i03 (last accessed 17.11.2016).

3 Bilimoria KY, Bentrem DJ, Lillemoe KD, Talamonti MS, Ko CY; Pancreatic Cancer Quality Indicator Development Expert Panel, American College of Surgeons: Assessment of pancreatic cancer care in the United States based on formally developed quality indicators. J Natl Cancer Inst 2009;101:848-859.

4 Kalish BT, Vollmer CM, Kent TS, Nealon WH, Tseng JF, Callery MP: Quality assessment in pancreatic surgery: what might tomorrow require? J Gastrointest Surg 2013;17:86-93; discussion 93.

5 Seufferlein T, Porzner M, Becker T, et al: S3-guideline exocrine pancreatic cancer (Article in German). Z Gastroenterol 2013;51:1395-1440.

6 Tempero MA, Arnoletti JP, Behrman SW, et al: Pancreatic adenocarcinoma, version 2.2012: featured updates to the NCCN Guidelines. J Natl Compr Cancer Netw 2012;10:703-713.

7 Hata T, Motoi F, Ishida M, et al: Effect of hospital volume on surgical outcomes after pancreaticoduodenectomy: a systematic review and meta-analysis. Ann Surg 2016;263:664-672.
8 Nimptsch U, Krautz C, Weber GF, Mansky T, Grützmann R: Nationwide in-hospital mortality following pancreatic surgery in Germany is higher than anticipated. Ann Surg 2016;264:1082-1090.

9 Buettner S, Gani F, Amini N, et al: The relative effect of hospital and surgeon volume on failure to rescue among patients undergoing liver resection for cancer. Surgery 2016;159:1004-1012.

10 Sahni NR, Dalton M, Cutler DM, Birkmeyer JD, Chandra A: Surgeon specialization and operative mortality in United States: retrospective analysis. BMJ 2016; 354:i3571.

11 Gooiker GA, Lemmens VE, Besselink MG, et al: Impact of centralization of pancreatic cancer surgery on resection rates and survival. Br J Surg 2014;101:1000-1005.

12 Gooiker GA, van der Geest LG, Wouters MW, et al: Quality improvement of pancreatic surgery by centralization in the western part of the Netherlands. Ann Surg Oncol 2011;18:1821-1829.

13 de Wilde RF, Besselink MG, van der Tweel I, et al: Impact of nationwide centralization of pancreaticoduodenectomy on hospital mortality. Br J Surg 2012;99:404-410.

14 Alsfasser G, Leicht H, Günster C, Rau BM, Schillinger G, Klar E: Volume-outcome relationship in pancreatic surgery. Br J Surg 2016;103:136-143.

15 Keck T, Wellner UF, Bahra M, et al: Pancreatogastrostomy versus pancreatojejunostomy for RECOnstruction after PANCreatoduodenectomy (RECOPANC, DRKS 00000767): perioperative and long-term results of a multicenter randomized controlled trial. Ann Surg 2016;263:440-449.
16 Mise Y, Vauthey J-N, Zimmitti G, et al: Ninety-day postoperative mortality is a legitimate measure of hepatopancreatobiliary surgical quality. Ann Surg 2015;262:1071-1078.

17 Witzigmann H, Diener MK, Kienkötter S, et al: No need for routine drainage after pancreatic head resection: the dual-center, randomized, controlled PANDRA trial (ISRCTN04937707). Ann Surg 2016;264: 528-537.

18 Dale W, Hemmerich J, Kamm A, et al: Geriatric assessment improves prediction of surgical outcomes in older adults undergoing pancreaticoduodenectomy: a prospective cohort study. Ann Surg 2014;259:960-965.

19 Roulin D, Cerantola Y, Demartines N, Schäfer M: Systematic review of delayed postoperative hemorrhage after pancreatic resection. J Gastrointest Surg 2011;15: 1055-1062.

20 Tol JA, Busch OR, van Delden OM, van Lienden KP, van Gulik TM, Gouma DJ: Shifting role of operative and nonoperative interventions in managing complications after pancreatoduodenectomy: what is the preferred intervention? Surgery 2014;156:622-631.

21 Wellner UF, Kulemann B, Lapshyn H, et al: Postpancreatectomy hemorrhage - incidence, treatment, and risk factors in over 1,000 pancreatic resections. J Gastrointest Surg 2014;18:464-475.

22 Clavien PA, Barkun J, de Oliveira ML, et al: The Clavien-Dindo classification of surgical complications: five-year experience. Ann Surg 2009;250:187-196. 